Article

\title{
Synthesis of Canthardin Sulfanilamides and Their Acid Anhydride Analogues via a Ring-Opening Reaction of Activated Aziridines and Their Associated Pharmacological Effects
}

\author{
Ling-Ling Chiang ${ }^{1,2}$, Ing-Jy Tseng ${ }^{3}$, Pen-Yuan Lin ${ }^{4}$, Shiow-Yunn Sheu ${ }^{4}$, Ching-Tung Lin ${ }^{5}$, \\ Yun-Han Hsieh ${ }^{4}$, Yi-Jing Lin ${ }^{4}$, Hsiao-Ling Chen ${ }^{4}$ and Mei-Hsiang Lin ${ }^{4, *}$ \\ Received: 3 December 2015; Accepted: 12 January 2016; Published: 16 January 2016 \\ Academic Editor: Wei-Zhu Zhong \\ 1 School of Respiratory Therapy, Taipei Medical University, Taipei 11031, Taiwan; llchiang@tmu.edu.tw \\ 2 Chest Medicine Department, Shuang-Ho Hospital, Taipei Medical University, Taipei 11031, Taiwan \\ 3 Gerontology Health Management, College of Nursing, Taipei Medical University, Taipei 11031, Taiwan; \\ Ingjy@tmu.edu.tw \\ 4 Department of Pharmaceutical Sciences, School of Pharmacy, Taipei Medical University, Taipei 11031, \\ Taiwan; lpy0620@tmu.edu.tw (P.-Y.L.); amel@tmu.edu.tw (S.-Y.S.); jay4273803@hotmail.com (Y.-H.H.); \\ m301101003@tmu.edu.tw (Y.-J.L.); m301101001@tmu.edu.tw (H.-L.C.) \\ 5 Department of Chemistry, Tam-Kang University, Danshui 25137, New Taipei City, Taiwan; \\ rocketqueenohya@hotmail.com \\ * Correspondence: mhl00001@tmu.edu.tw; Tel.: +886-2-2736-1661 (ext. 6138)
}

\begin{abstract}
The cantharidinimide derivatives, $\mathbf{5 a}-\mathbf{h}$, including sulfanilamides containing pyrimidyl, pyrazinyl, hydrogen, thiazolyl, and oxazolyl groups were synthesized. Modification of cantharidinimide by means of the reaction of activated aziridine ring opening led to the discovery of a novel class of antitumor compounds. The analogues $10 \mathbf{i}-\mathbf{k}, \mathbf{1 1 1}-\mathbf{n}, \mathbf{1 2 0}-\mathbf{p}$, and $\mathbf{1 6 q}-\mathbf{s}$ were obtained from treating cantharidinimide 6 and analogues $(7,8$, and 13) with activated aziridines, which produced a series of ring-opened products including normal and abnormal types. Some of these compounds showed cytotoxic effects in vitro against HL-60, Hep3B, MCF7, and MDA-MB-231 cancer cells. The most potent cytostatic compound, $N$-cantharidinimido-sulfamethazine (5a), exhibited anti-HL-60 and anti-Hep3B cell activities. Two compounds $5 \mathrm{~g}$ and $\mathbf{5 h}$ displayed slight effects on the Hep3B cell line, while the other compounds produced no response in these four cell lines.
\end{abstract}

Keywords: cantharidin; cantharidinimide; methylsulfanylaziridine

\section{Introduction}

Cantharidin 1 (Figure 1), isolated from Mylabris caraganae and various other insects, is a terpenoid and has a high vesicant potency [1]. It is used to treat piles, warts, ulcers, and molluscum through topical application and is also used as an abortifacient and aphrodisiac [1,2]. In clinical studies, it had significant activity against liver tumors and the KB cell line in tissue culture at low concentrations $[3,4]$. The clinical application of cantharidin is limited by its cytotoxic properties [5,6]. It is an inhibitor of the serine/threonine protein phosphatases, PP1 and PP2A, leading to multiple cellular effects such as DNA damage, cell cycle arrest, and apoptosis [7-10]. Cantharidin induces apoptosis in many human cancer cells such as pancreatic carcinoma [9], colon cancer [10], lung cancer [11], melanomas [12], and bladder cancer [13]. It also induces endoplasmic reticular (ER) stress and autophagic cell death [13-15]. Recently, certain reports indicated that $N$-cantharidin derivatives have antitumor and antihepatoma properties [16-19]. Some cantharidinimides showed tumor-inhibitory action in animals, and several 
cantharidinimides exhibited inhibitory effects against xanthine oxidase [20]. A number of modified cantharidin analogues were synthesized and their anticancer activities against a wide range of human tumor cell lines were evaluated [21-27].

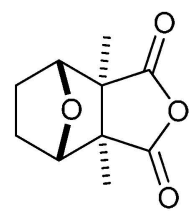

1: cantharidin

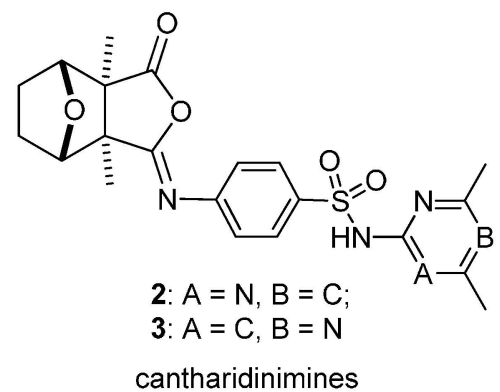

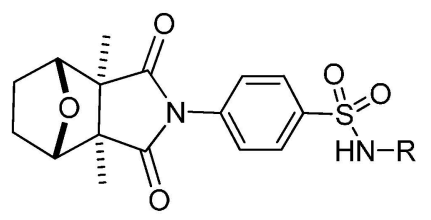

cantharidin-sulfanilamides

Figure 1. Structures of cantharidin (1), cantharidinimines $(2,3)$, and cantharidin-sulfanilamides.

In our previous research, we synthesized many cantharidinimides by modification with alkyl, phenyl, pyridinyl, thiazolyl, and thiadiazolyl groups [28-30]. Those compounds showed certain degrees of cytotoxic activities against HL-60 (human myeloid leukemia cells), Hep 3B and Hep G2 (human hepatocarcinoma cells), NUGC (human gastric carcinoma cells), and HONE-1 (human nasopharyngeal carcinoma cells). Cantharidinimides with better solubility showed greater cytotoxicity against human hepatoma cell lines. The cantharidinimides with an electron-withdrawing group $\left(\mathrm{R}_{1}\right)$ on the aromatic ring $(\mathrm{X})$ exhibited increased cytotoxicity to cancer cell lines. Then, we used this feature and synthesized different imines to study their cytotoxic effects [31]. These results showed that cantharidinimine derivatives had greater cytotoxic effects than cantharidinimides. Especially, cantharidinimine derivative 2 (Figure 1), which has a sulfanyl group, exhibited a $50 \%$ inhibitory concentration $\left(\mathrm{IC}_{50}\right)$ value of $6 \mu \mathrm{M}$ against HL-60 cells as did cantharidin $\left(\mathrm{IC}_{50}\right.$ value of $\left.7.2 \mu \mathrm{M}\right)$.

However, cantharidinimides and their analogues also display inhibition of the inducible nitric oxide (NO) synthase (iNOS) pathway, and they inhibited NO synthase activity by more than $90 \%$. Thus, they might be advantageous for anticancer therapy $[32,33]$. Because of this, we synthesized canthardin sulfanilamides (Figure 1) and tested their cytotoxic effects on human cancer cell lines (HL-60 and Hep3B), human breast carcinoma cells (MCF7), and human breast carcinoma cells (MDA-MB-231) since incidence rates occur rapidly and more widely among Asian peoples than in Westerners. Growth inhibition was evaluated using an MTT assay. In order to find new cantharidinimides and related imides containing the sulfonamide group, we synthesized sulfonamido-ethyldicarboximide derivatives $(\mathbf{1 0 i}-\mathbf{k}, \mathbf{1 1 1}-\mathbf{n}, \mathbf{1 2 0}-\mathbf{p}$, and $\mathbf{1 6 q}-\mathbf{s})$ and tested their cytotoxic activities.

\section{Results and Discussion}

\subsection{Chemistry}

We prepared effective cantharidin sulfanilamides by heating the reactant cantharidin (1) and primary arylamines of sulfanilamide derivatives $(\mathbf{4 a}-\mathbf{h})$ to $c a .200{ }^{\circ} \mathrm{C}$ with $3 \mathrm{~mL}$ of dry toluene and 1-2 $\mathrm{mL}$ of TEA in a high-pressure sealed tube (Buchi glasuster 0032) to provide compounds $\mathbf{5 a}-\mathbf{h}$ in good yields (Scheme 1). The sulfonamide derivatives containing pyrimidine, pyrazine, hydrogen, thiazole, and oxazole were sulfadiazine, sulfamethazine, sulfisomidine, sulfamerazine, sulfaquinoxaline, sulfacarbamide, 2-thiazolyl-sulfanilamide, and sulfamethoxazole (4a-h). Yields varied $2 \%-62 \%$ and showed a trend compatible with the expected basicity, and the characters of the bulky quinoxaline and the more-basic carbamide groups influenced compounds $5 \mathrm{~d}$ ( $6 \%$ yield) and $\mathbf{5 e}(2 \%$ yield). The results obtained with $\mathbf{5 a}-\mathbf{c}$ and $\mathbf{5} \mathbf{f}-\mathbf{h}$, however, showed the same strong electron-withdrawing sulfonyl $\left(\mathrm{SO}_{2}\right)$ group and aromatic amino $\left(\mathrm{NH}_{2}\right)$ basicity, which were unknown but really influenced the product yields, and the characters of a long side chain with resonance and induction effects were considered spontaneously. 


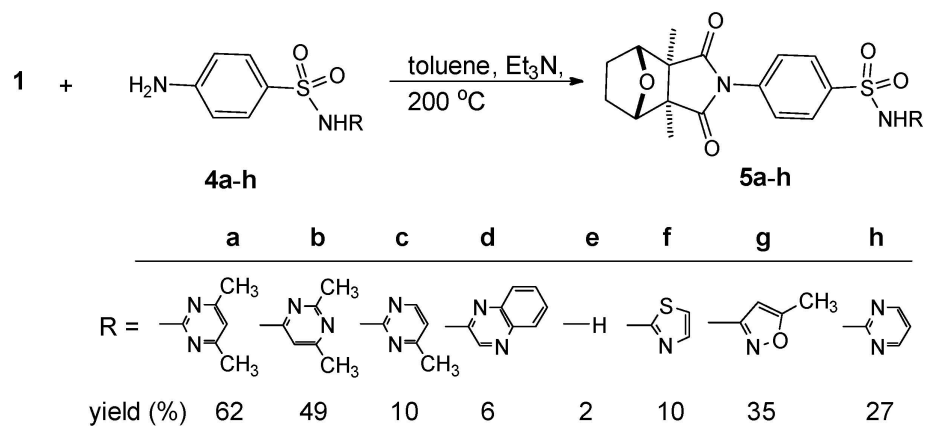

Scheme 1. Synthesis of cantharidin-sulfanilamides $\mathbf{5 a - h}$.

In order to find some new cantharidinimides, cantharidin was treated with ammonia and yielded cantharidinimide (6) which was reacted with dimethyl or monomethyl-activated aziridins. Thus, 6 and its imide derivatives ( 7 and $\mathbf{8}$ ) were reacted with $N, N^{\prime}$-dimethylformamide (DMF) in a sealed tube under reflux for $3 \mathrm{~h}$, after which it was cooled, and then activated aziridine (9) was added dropwise and refluxed for another $2 \mathrm{~h}$ to yield compounds 10i-k, 111-n, and 12o-p (Scheme 2). In this study, the ring opening reaction of $\mathrm{N}$-sulfonyl 2,2-dimethylaziridine by imide nucleophiles (Figure 2a,d,e), which attacked the unsubstituted carbon, was detected. However, the ring-opening reaction of $N$-acyl 2,2-dimethylaziridine by various nucleophiles in the absence of an acid was reported to proceed with a formal attack on the tertiary carbon of the aziridine ring (abnormal opening) [34]. No products of a "normal opening" (in the $\mathrm{S}_{\mathrm{N}} 2$ sense) were observed when the R groups were phenyl or biphenyl (Figure 2). The small amount of normal opening yield (when $\mathrm{R}$ was phenyl) was possibly the result of a displacement in the inversional ground states. It was through the regioselectivity ring-opening reaction, and the strong preference for normal-opening sulfonyl activation ( $\mathrm{R}$ group was tosyl) that simply reflected the steric hindrance of a nucleophilic attack on the tertiary aziridine carbon in the inversional ground state. A single electron transfer (SET) mechanism was proposed to account for this unexpected behavior [35,36]. Therefore, we proposed that both normal and abnormal opening reactions of activated aziridines were slowed down compared to a reaction in the absence of the two methyl groups, the former clearly more so than the latter, and the white crystalline compounds $\mathbf{1 0} \mathbf{i}-\mathbf{k}$ were obtained (Scheme 2). The same method was used with cantharidin analogue compounds to obtain products $\mathbf{1 1 1}-\mathbf{n}$ and $\mathbf{1 2 0}-\mathbf{p}$.

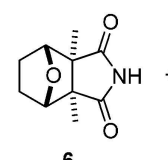<smiles></smiles>

10j: $R_{1}=H, R_{2}=\mathrm{CH}_{3},(21 \%$ 10k: $\mathrm{R}_{1}=\mathrm{CH}_{3}, \mathrm{R}_{2}=\mathrm{H},(22 \%)$
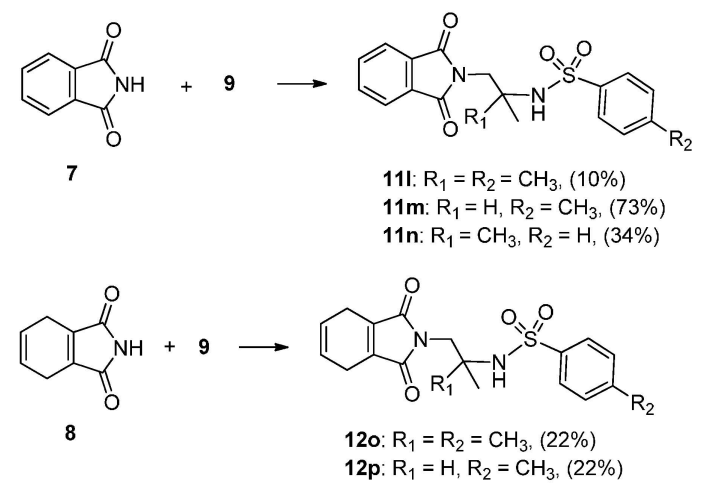

Scheme 2. Synthesis of compounds $10 \mathbf{i}-\mathbf{k}, \mathbf{1 1 1}-\mathbf{n}$, and 12o-p. 
Normal opening of $N$-sulfonyl $N$-methylaziridine<smiles>[R]S(=O)(=O)NC(C)CNCC#N</smiles>

planarization effect devoid of resonance contribution nucleophilic ring opening depends on activation<smiles>C[C@]12C(=O)NC(=O)[C@@]1(C)[C@H]1CC[C@H]2O1</smiles>

a

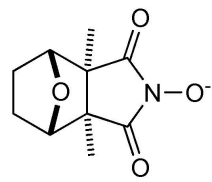

b<smiles>C[C@]12C(=O)N(O)C(=O)[C@@]1(C)[C@H]1C=C[C@H]2O1</smiles>

C<smiles>O=C1NC(=O)c2ccccc21</smiles>

d<smiles>O=C1NC(=O)C2=C1CC=CC2</smiles>

e

Abnormal opening of $\mathrm{N}$-acyl $\mathrm{N}$-methylaziridine

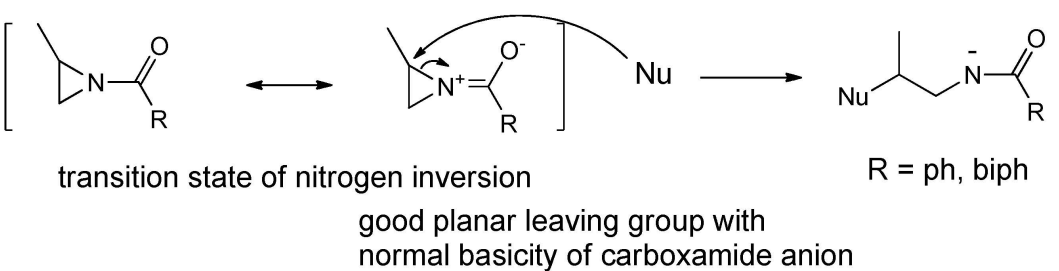

Figure 2. Regioselectivity in nucleophilic ring opening of $N$-sulfony and $N$-acyl activated 2-methylaziridine.

Structures of products $10 \mathbf{j}$ and $10 \mathrm{i}$ followed from the ${ }^{1} \mathrm{H}-\mathrm{NMR}$ spectra of chromatographic fractions, and the main evidence for $\mathbf{A}$ included signals of cantharidinimide's $\mathrm{CH}_{3}$ (position 8 and 9), $\mathrm{NCH}_{2}$ (position 10), and $\mathrm{OCH}$ (position 4 and 7) protons at 1.22 (singlet), 3.51 (singlet), and $4.54 \mathrm{ppm}$ (singlet), respectively (Table 1, Figure 3). These signals indicated the same values of the protons on two kinds of methyl, $\mathrm{OCH}$, and $\mathrm{NCH}_{2}$ groups of product $10 \mathrm{i}$ which might have greater steric hindrance (compared to $\mathbf{1 0 j}$, Figure $3 \mathrm{~B}$ ) repulsion between the two pairs of methyl groups, and the conformation for 10i might have the least electrostatic repulsion between the proton of nitrogen and the two oxygen atoms of cantharidinimide with their negative partial charge. A downfield shift of product $10 \mathrm{j}$ relative to $10 \mathrm{i}$ was observed for these protons. These shifts and coupling constants (Table 1) became complicated for several possible reasons: the hydrogen bonding bridge enhanced the electron-withdrawing capacity of the sulfonyl group and shifted the signals downfield from the usual range, and the three methyl groups might have less steric hindrance to each other. Thus, the structure became the conformationally fixed type B (Figure 3).

Table 1. ${ }^{1} \mathrm{H}$ chemical shifts $\left({ }^{\mathrm{TM}}\right)$ of sulfonamide-cantharidinimides $10 \mathbf{i}$ and $\mathbf{1 0 j}\left(\mathrm{CDCl}_{3}, 500 \mathrm{MHz}\right)$.

\begin{tabular}{ccc}
\hline \multirow{2}{*}{ Position } & $\mathbf{1 0 \mathbf { i }}$ & $\mathbf{1 0 j}$ \\
\cline { 2 - 3 } & $\delta_{\mathbf{H}}(\boldsymbol{J}$ in Hz) & $\delta_{\mathbf{H}}(\boldsymbol{J} \mathbf{~ i n ~ H z})$ \\
\hline 4,7 & $4.54(\mathrm{~s})$ & $4.55(\mathrm{~s})$ \\
5,6 & $1.68-1.70(\mathrm{~m}) ; 1.79-1.81(\mathrm{~m})$ & $1.67-1.70(\mathrm{~m}) ; 1.79-1.83(\mathrm{~m})$ \\
8,9 & $1.22(\mathrm{~s})$ & $1.21(\mathrm{~s})$ \\
10 & $3.51(\mathrm{~s})$ & $3.38(\mathrm{dd}, 3.8,3.8) ; 3.61-3.64(\mathrm{~m})$ \\
11 & - & $3.52(\mathrm{dd}, 9.7,9.7)$ \\
12 & $5.77(\mathrm{~s})$ & $4.68(\mathrm{~d}, 9)$ \\
15,19 & $7.72(\mathrm{~d}, 8)$ & $7.70(\mathrm{~d}, 8)$ \\
16,18 & $7.23(\mathrm{~d}, 8)$ & $7.27(\mathrm{~d}, 8)$ \\
20 & $2.39(\mathrm{~s})$ & $2.41(\mathrm{~s})$ \\
21 & $1.14(\mathrm{~s})$ & $0.93(\mathrm{~d}, 6.7)$ \\
\hline
\end{tabular}




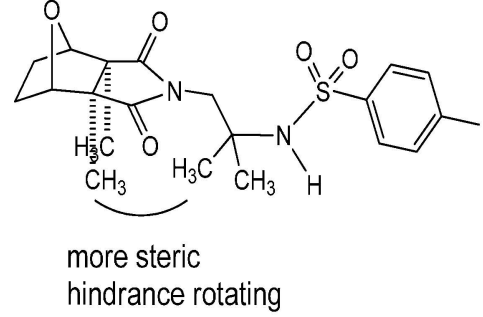

A (10i)

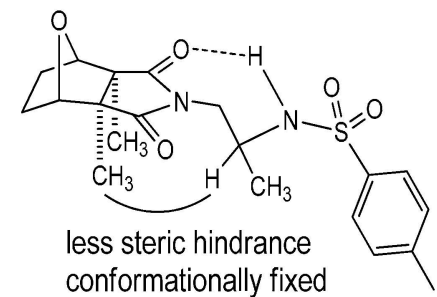

$B(10 \mathrm{j})$

Figure 3. Conformation of $\mathbf{1 0} \mathbf{i}$ and $\mathbf{1 0} \mathbf{j}$.

The $N$-pyrimidyl, $N$-thiazolyl, $N$-oxazolylsulfonylcantharidinimides, $\mathbf{5 a}-\mathbf{h}$, were prepared by means of a pressure technique synthesis, and their analogous derivatives $(\mathbf{1 0} \mathbf{i}-\mathbf{k}, \mathbf{1 1 1}-\mathbf{m}$, and $\mathbf{1 2 0}-\mathbf{p})$ were synthesized via nucleophilic ring opening of methyl- or dimethylbenzenesulfonylaziridine and methyl- or dimethyltoluenesulfonylaziridine, after reacting with several analogous nucleophilic bases. Compounds 16q-s were obtained from $N$-hydroxylimide 13 and activated aziridine by treatment with $\mathrm{NaH}$ base in dry THF for $24 \mathrm{~h}$ (Scheme 3). After evaporation, it was purified by silica gel column chromatography and recrystallized in methanol. From these reactions, it should be noted that the characters of various bases (Figure 2a-e) strongly confirmed the influence of the positions of the ring opening of the activated aziridines. The preparative technique, the temperature, the drying solvent, and the formation of nucleophilic bases were also influenced by other factors that can cause strong variations in the results. The reaction steps and time were also crucial factors in this formation. The results of these yields strongly confirmed the influence of the sulfanilamide side chain $(5 \mathbf{e}, 2 \%, \mathrm{R}=\mathrm{H})$.<smiles>O=C1C2C3C=CC(O3)C2C(=O)N1O</smiles>

13<smiles>CC1CN1S(=O)(=O)c1ccccc1</smiles>

14

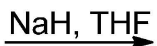<smiles>[R7]C(NS(=O)(=O)c1ccccc1)C([R2])ON1C(=O)C2C3C=CC(O3)C2C1=O</smiles>

16q: $\mathrm{R}_{1}=\mathrm{CH}_{3}, \mathrm{R}_{2}=\mathrm{H},(13 \%)$ 16r: $\mathrm{R}_{1}=\mathrm{H}, \mathrm{R}_{2}=\mathrm{CH}_{3},(9 \%)$<smiles>O=C1C2C3C=CC(O3)C2C(=O)N1OCCNS(=O)(=O)c1cc(Cl)ccc1Cl</smiles>

$16 \mathrm{~s}(48 \%)$

\section{$\stackrel{\mathrm{NaH}, \mathrm{THF}}{\longrightarrow}$}

Scheme 3. Synthesis of compounds $\mathbf{1 6 q}-\mathbf{s}$.

\subsection{Pharmacology}

Cytotoxicities of the cantharidin-sulfanilamide derivatives, $\mathbf{5 a}-\mathbf{h}$, were tested by suppressing the growth of the HL-60, Hep3B, MCF7, and MDA-MB-231 human carcinoma cells using MTT cell viability assays. Some compounds showed inhibitory effects on HL-60 and Hep3B cells. Cantharidin 1 was more toxic and exhibited greater cytotoxicity, while the synthesized cantharidinimides were less toxic but also exhibited inhibitory effects (Table 2). In this study, compound $\mathbf{5 a}$, which had two methyl groups on the pyrimidyl ring, displayed strong inhibitory effects on the HL-60 and Hep3B cell lines, as did cantharidin. $N$-Cantharidinimido-sulfamethazine $5 \mathbf{a}$ was less toxic compared to $\mathrm{N}$-cantharidinimino-sulfamethazine 2 against HL-60. This result confirmed our previous study. 
Compounds $\mathbf{5 b}$ and $\mathbf{5} \mathbf{c}$ also had methyl groups, but, unfortunately, no activities were detected against either of the two cell lines as compounds $\mathbf{5} \mathbf{h}$ and $\mathbf{5 d}$, thus the methyl group positions highly influenced the results. Compound $\mathbf{5 g}$ with an oxazolyl group exhibited medium cytotoxicity against the Hep3B cell line, but compound $\mathbf{5 f}$, although with a thiazolyl group, displayed no inhibitory effects on either cell line. Aziridine side chains contained imide derivatives, and analogues 10i-k, 111-n, 12o-p, and 16q-s exhibited weak inhibitory effects against the Hep3B cell line but displayed very low effects against the HL-60, MCF7, and MDA-MB-231 cell lines.

Table 2. Cytotoxicity effect of $\mathbf{1}$, cantharidin-sulfanilamides (5a-h), and imide analogues (10i-k, 111-n, 12o-p, and 16q-s) in human hepatocellular carcinoma (Hep3B), myeloid leukemia (HL-60), and human breast adenocarcinoma (MCF7 and MDA-MB-231) cell lines.

\begin{tabular}{ccccc}
\hline \multirow{2}{*}{$\mathbf{p d ~ N o .}$} & \multicolumn{4}{c}{$\mathbf{I C}_{\mathbf{5 0}}(\boldsymbol{\mu} \mathbf{M})^{\mathbf{a}}$} \\
\cline { 2 - 5 } & $\mathbf{H L}-\mathbf{6 0}$ & Hep3B & MCF7 & MDA-MB-231 \\
\hline $\mathbf{1}$ & 5.6 & 3.4 & - & - \\
$\mathbf{5 a}$ & 38.2 & 9 & $>100$ & $>100$ \\
$\mathbf{5 b}$ & $>100$ & $>100$ & $>100$ & $>100$ \\
$\mathbf{5} \mathbf{c}$ & $>100$ & $>100$ & $>100$ & $>100$ \\
$\mathbf{5 d}$ & $>100$ & $>100$ & $>100$ & $>100$ \\
$\mathbf{5 e}$ & $>100$ & $>100$ & $>100$ & $>100$ \\
$\mathbf{5 f}$ & $>100$ & $>100$ & $>100$ & $>100$ \\
$\mathbf{5 g}$ & $>100$ & 17 & $>100$ & $>100$ \\
$\mathbf{5 h}$ & $>100$ & 80 & $>100$ & $>100$ \\
$\mathbf{1 0} \mathbf{1 0}$ & $>100$ & $>100$ & $>100$ & $>100$ \\
$\mathbf{1 0}$ & $>100$ & $>100$ & $>100$ & $>100$ \\
$\mathbf{1 0 k}$ & $>100$ & $>100$ & $>100$ & $>100$ \\
$\mathbf{1 1 1}$ & $>100$ & $>100$ & $>100$ & $>100$ \\
$\mathbf{1 1} \mathbf{1 1 n}$ & $>100$ & $>100$ & $>100$ & $>100$ \\
$\mathbf{1 2}$ & $>100$ & $>100$ & $>100$ & $>100$ \\
$\mathbf{1 2} \mathbf{p}$ & $>100$ & $>100$ & $>100$ & $>100$ \\
$\mathbf{1 6 q}$ & $>100$ & $>100$ & $>100$ & $>100$ \\
$\mathbf{1 6 r}$ & $>100$ & $>100$ & $>100$ & $>100$ \\
$\mathbf{1 6 s}$ & $>100$ & $>100$ & $>100$ & $>100$ \\
\hline
\end{tabular}

${ }^{\mathrm{a}} \mathrm{IC}_{50}$ was calculated after $48 \mathrm{~h}$ of continuous drug exposure, values are the mean of three to four experiments with coefficients of variation of $5 \%-10 \%$.

The cytotoxicity of cantharidin was investigated in both the NCI and Oncotest cell line panels [37]. It showed profound activity at low micromolar concentrations ( $\log _{10} \mathrm{IC}_{50}$ values of -6.980 to $-5.009 \mathrm{M}$ ), and revealed extreme cytotoxicity toward cell lines of diverse tumor types. According to a COMPARE analysis [38] of microarray-based transcriptome-wide mRNA expressions, 21 genes were found to be significantly correlated with the response of 60 tumor cell lines to cantharidin. Some researchers screened their synthetic compounds against human cancer cell lines and used a COMPARE analysis to evaluate the anticancer activity and predict the probable mechanism [27,39-42]. Therefore, the following studies examined the possible mechanism using a standard NCI-60 test and COMPARE analysis of $\mathbf{5 a}$.

\section{Experimental Section}

\subsection{Synthesis}

\subsubsection{General Experimental Procedures}

General procedures were followed for the reaction of ammonia with cantharidine and the other acid anhydride. These compounds were prepared according to similar procedures, and the reactions took place in high-pressure tubes (Büchi Glasuster 0032, Zürich, Switzerland). Cantharidin or acid 
anhydrides were added to a tube containing $3 \mathrm{~mL}$ of dry toluene and triethylamine (TEA), and the solution was stirred and heated to $c a .200{ }^{\circ} \mathrm{C}$. After being stirred for $2 \mathrm{~h}$, the mixture was evaporated, and the residual mass was purified by column chromatography on silica gel and recrystallized from methanol.

All temperatures are reported in degrees centigrade. Melting points were determined with a Büchi B-545 melting point apparatus (Büchi, Flawil, Switzerland) and were uncorrected. $[\alpha]_{\mathrm{D}}^{20}$ values were recorded on a JASCO P-1020 Digital Polarimeter (JASCO, Tokyo, Japan). Infrared spectra were recorded on a Perkin-Elmer Model 882 (Thermo Fisher Scientific Inc., New York, NY, USA) and Nicolet 510 pet and Thermo Mattson IR 300 (Thermo Fisher Scientific Inc.) spectrophotometers. ${ }^{1} \mathrm{H}$-nuclear magnetic resonance (NMR) spectra (in $\mathrm{CDCl}_{3}$ unless otherwise stated) were recorded at $500 \mathrm{MHz}$ on a Bruker Advance DRX and AM-500 FT (Bruker Co., Bremen, Germany). Mass spectra were obtained on a JOEL JMSHX 110 FAB-MS (Joel, Tokyo, Japan) spectrometer and MAT-95XL HRMS (Finnigan, München, Germany).

\subsubsection{Test Samples}

Chinese blister beetles were extracted with a water-ethanol (1:1) solution, filtered through celite, purified by chromatography on silica gel, and then recrystallized with ethanol to give cantharidin 1 . Compounds $\mathbf{5 a}-\mathbf{h}, \mathbf{1 0} \mathbf{i}-\mathbf{k}, \mathbf{1 1 1}-\mathbf{n}, \mathbf{1 2 0}-\mathbf{p}$, and $\mathbf{1 6 q}-\mathbf{s}$ were prepared from cantharidin, acid anhydride, and primary amines in the presence of TEA in toluene in high-pressure tubes. The mass spectra of all compounds were measured, and ${ }^{1} \mathrm{H}-\mathrm{NMR}$ was also used for testing.

\subsubsection{Physical Data of Cantharidinimide Derivatives and Analogues}

N-Cantharidinimido-sulfamethazine (5a): 62\% yield, m.p. $195-196{ }^{\circ} \mathrm{C}$. IR (KBr): 1709 (amide) cm ${ }^{-1}$; ${ }^{1} \mathrm{H}-\mathrm{NMR}: \delta 1.25(6 \mathrm{H}, \mathrm{s}), 1.75-1.77(2 \mathrm{H}, \mathrm{m}), 1.86-1.87(2 \mathrm{H}, \mathrm{m}), 2.05(6 \mathrm{H}, \mathrm{s}), 4.69(2 \mathrm{H}, \mathrm{t}, J=2.3,2.6 \mathrm{~Hz})$, $6.64(1 \mathrm{H}, \mathrm{s}$, pyrimidinyl $\mathrm{H}), 7.52(2 \mathrm{H}, \mathrm{d}, J=8.6 \mathrm{~Hz}$, phenyl $\mathrm{H}), 8.26(2 \mathrm{H}, \mathrm{d}, J=8.7 \mathrm{~Hz}$, phenyl $\mathrm{H})$; FAB-MS $m / z$ (rel. int.): 457 [M + H] ${ }^{+}(100), 154,124$; HRMS, calcd. for $\mathrm{C}_{22} \mathrm{H}_{25} \mathrm{~N}_{4} \mathrm{O}_{5} \mathrm{~S}[\mathrm{M}+\mathrm{H}]^{+} 457.1546$, found 457.1547 .

N-Cantharidinimido-sulfisomidine (5b): 49\% yield, m.p. $163-164{ }^{\circ} \mathrm{C}$. IR (KBr): 1710 (amide) $\mathrm{cm}^{-1}$; ${ }^{1} \mathrm{H}-\mathrm{NMR}: \delta 1.26(6 \mathrm{H}, \mathrm{s}), 1.75-1.77(2 \mathrm{H}, \mathrm{m}), 1.78-1.80(2 \mathrm{H}, \mathrm{m}), 2.54(3 \mathrm{H}, \mathrm{s}), 2.67(3 \mathrm{H}, \mathrm{s}), 4.69(2 \mathrm{H}, \mathrm{t}$, $J=2.3 \mathrm{~Hz}), 6.9(1 \mathrm{H}$, s, pyrimidinyl $\mathrm{H}), 7.55(2 \mathrm{H}, \mathrm{d}, J=8.4 \mathrm{~Hz}$, phenyl $\mathrm{H}), 8.06(2 \mathrm{H}, \mathrm{d}, J=8.4 \mathrm{~Hz}$, phenyl H); FAB-MS m/z (rel. int.) $457[\mathrm{M}+\mathrm{H}]^{+}(22), 279,154$; HRMS $\left(\mathrm{FAB}^{+}\right.$), calcd. for $\mathrm{C}_{20} \mathrm{H}_{22} \mathrm{~N}_{3} \mathrm{O}_{6} \mathrm{~S} 457.1546$, found 457.1549 .

N-Cantharidinimido-sulfamerazine (5c): $10 \%$ yield, m.p. $254-256{ }^{\circ} \mathrm{C} . \mathrm{IR}(\mathrm{KBr}): 1710$ (amide) $\mathrm{cm}^{-1}$; ${ }^{1} \mathrm{H}-\mathrm{NMR}: \delta 1.25(6 \mathrm{H}, \mathrm{s}), 1.74-1.76(2 \mathrm{H}, \mathrm{m}), 1.84-1.87(2 \mathrm{H}, \mathrm{m}), 2.42(3 \mathrm{H}, \mathrm{s}), 4.68(2 \mathrm{H}, \mathrm{t}, J=2.3 \mathrm{~Hz})$, $6.80(1 \mathrm{H}, \mathrm{d}, J=5.2 \mathrm{~Hz}$, pyrimidinyl $\mathrm{H}), 7.52(2 \mathrm{H}, \mathrm{d}, J=8.5 \mathrm{~Hz}$, phenyl $\mathrm{H}), 8.24(2 \mathrm{H}, \mathrm{d}, J=8.6 \mathrm{~Hz}$, phenyl $\mathrm{H}), 8.42(1 \mathrm{H}, \mathrm{d}, J=5.1 \mathrm{~Hz}$, pyrimidinyl $\mathrm{H})$; $\mathrm{HRMS}\left(\mathrm{FAB}^{+}\right)$, calcd for $\mathrm{C}_{21} \mathrm{H}_{23} \mathrm{~N}_{4} \mathrm{O}_{5} \mathrm{~S} 443.1311$, found 443.1313 .

N-Cantharidinimido-sulfaquinoxaline (5d): 6\% yield, m.p. $253-255^{\circ} \mathrm{C} . \mathrm{IR}(\mathrm{KBr}): 1704$ (amide) $\mathrm{cm}^{-1}$; ${ }^{1} \mathrm{H}-\mathrm{NMR}: \delta 1.20(6 \mathrm{H}, \mathrm{s}), 1.67-1.71(2 \mathrm{H}, \mathrm{m}), 1.80-1.84(2 \mathrm{H}, \mathrm{m}), 4.63(2 \mathrm{H}, \mathrm{t}, J=2.8,2.4 \mathrm{~Hz}), 7.45-7.48$ $\left(3 \mathrm{H}, \mathrm{m}, \mathrm{H}-6^{\prime}\right.$ peak overlap, $\left.J=8.5 \mathrm{~Hz}\right), 7.58(2 \mathrm{H}, \mathrm{t}-\mathrm{like}, 2 \mathrm{H}), 7.89\left(1 \mathrm{H}, \mathrm{d}, J=8.2 \mathrm{~Hz}, \mathrm{H}-8^{\prime}\right), 8.14(2 \mathrm{H}, \mathrm{d}$, $J=8.6 \mathrm{~Hz}), 8.59(1 \mathrm{H}, \mathrm{s})$. HRMS $\left(\mathrm{FAB}^{+}\right)$, calcd. for $\mathrm{C}_{24} \mathrm{H}_{22} \mathrm{~N}_{4} \mathrm{O}_{5} \mathrm{~S} 478.1305$, found 478.1307 .

$\mathrm{N}$-Cantharidinimido-sulfacarbamide (5e): $2 \%$ yield, m.p. $115-116^{\circ} \mathrm{C}$. IR (KBr): 1776, 1706 (amide) cm ${ }^{-1}$; ${ }^{1} \mathrm{H}-\mathrm{NMR}\left(\mathrm{DMSO}-\mathrm{d}_{6}\right): \delta 1.28(6 \mathrm{H}, \mathrm{s}), 1.69-1.70(2 \mathrm{H}, \mathrm{m}), 1.95-1.96(2 \mathrm{H}, \mathrm{m}), 4.63(2 \mathrm{H}, \mathrm{t}-\mathrm{like}), 7.54(2 \mathrm{H}, \mathrm{d}$, $J=8.6 \mathrm{~Hz}), 8.02(2 \mathrm{H}, \mathrm{d}, J=8.4 \mathrm{~Hz})$; HRMS, calcd. for $\mathrm{C}_{16} \mathrm{H}_{18} \mathrm{~N}_{2} \mathrm{O}_{5} \mathrm{~S} 350.0894$, found 350.0895.

$\mathrm{N}$-Cantharidinimido-N'-(2-thiazolyl)-sulfanilamide (5f): $10 \%$ yield, m.p. $125-127^{\circ} \mathrm{C}$. IR (KBr): 1706 (amide) $\mathrm{cm}^{-1}$; ${ }^{1} \mathrm{H}-\mathrm{NMR}: \delta 1.25(6 \mathrm{H}, \mathrm{s}), 1.74-1.76(2 \mathrm{H}, \mathrm{m}), 1.85-1.87(2 \mathrm{H}, \mathrm{m}), 4.68(2 \mathrm{H}, \mathrm{s}), 6.54(1 \mathrm{H}, \mathrm{d}, J=4.5 \mathrm{~Hz}$, thiazolyl N-CH), $7.12(1 \mathrm{H}, \mathrm{d}, J=4.5 \mathrm{~Hz}$, thiazolyl S-CH), $7.47(2 \mathrm{H}, \mathrm{d}, J=8.2 \mathrm{~Hz}$, phenyl H), $8.02(2 \mathrm{H}$, 
$\mathrm{d}, J=8.5 \mathrm{~Hz}$, phenyl H); FAB-MS m/z (rel, int): $434[\mathrm{M}+\mathrm{H}]^{+}$(50), 154 (100); HRMS, calcd. for $\mathrm{C}_{19} \mathrm{H}_{20} \mathrm{~N}_{3} \mathrm{O}_{5} \mathrm{~S}_{2} 434.0844$, found 434.0847 .

N-Cantharidinimido-sulfamethoxazole (5g): 35\% yield; m.p. $211-213{ }^{\circ} \mathrm{C}$. IR (KBr): $1710(\mathrm{C}=\mathrm{O}) \mathrm{cm}^{-1}$; ${ }^{1} \mathrm{H}-\mathrm{NMR}$ (acetone- $\left.d_{6}\right): \delta 1.58(6 \mathrm{H}, \mathrm{s}), 2.25-2.26(4 \mathrm{H}, \mathrm{m}), 5.02(2 \mathrm{H}, \mathrm{t}, J=2.8 \mathrm{~Hz}), 2.78(3 \mathrm{H}, \mathrm{s}), 6.69(1 \mathrm{H}, \mathrm{s})$, $8.01(2 \mathrm{H}, \mathrm{t}, J=6.9 \mathrm{~Hz}), 8.48(2 \mathrm{H}, \mathrm{d}, J=6.7,1.9 \mathrm{~Hz}) ;$ FAB-MS $\mathrm{m} / \mathrm{z}$ (rel, int): 432 [M + H] ${ }^{+}(100)$; HRMS calcd. for $\mathrm{C}_{20} \mathrm{H}_{22} \mathrm{~N}_{3} \mathrm{O}_{6} \mathrm{~S} 432.1229$, found 432.1226 .

$\mathrm{N}$-Cantharidinimido-sulfadiazine (5h): $27 \%$ yield; $262-263{ }^{\circ} \mathrm{C}$; IR (KBr): 1710 (amide) $\mathrm{cm}^{-1} ;{ }^{1} \mathrm{H}-\mathrm{NMR}$ (acetone- $\left.d_{6}\right): \delta 1.27(6 \mathrm{H}, \mathrm{s}), 1.64-1.66(4 \mathrm{H}, \mathrm{m}), 4.57(2 \mathrm{H}, \mathrm{t}, J=2.8 \mathrm{~Hz}), 7.06(1 \mathrm{H}, \mathrm{t}, J=4.7 \mathrm{~Hz}), 7.54(2 \mathrm{H}, \mathrm{t}$, $J=4.3 \mathrm{~Hz}), 8.23(2 \mathrm{H}, \mathrm{t}, J=4.3 \mathrm{~Hz}), 8.50(2 \mathrm{H}, \mathrm{d}, J=5.0 \mathrm{~Hz}) ;$ FAB-MS $m / z$ (rel, int) $429[\mathrm{M}+\mathrm{H}]^{+}(100)$; HRMS calcd. for $\mathrm{C}_{20} \mathrm{H}_{21} \mathrm{~N}_{4} \mathrm{O}_{5} \mathrm{~S} 429.1233$, found 429.1229 .

N-(2,2-Dimethylethyl-p-toluolsulfonamide)cantharidinimide (10i): $14 \%$ yield, $154-155^{\circ} \mathrm{C}$. IR (KBr): 1147 $\left(\mathrm{S}(=\mathrm{O})_{2}\right), 1401\left(\mathrm{~S}(=\mathrm{O})_{2}\right), 1691(\mathrm{C}=\mathrm{O}), 3264(\mathrm{NH}) \mathrm{cm}^{-1} ;{ }^{1} \mathrm{H}-\mathrm{NMR}$ (see Table 1); HRMS, calcd. for $\mathrm{C}_{21} \mathrm{H}_{28} \mathrm{~N}_{2} \mathrm{O}_{5} \mathrm{~S} 420.1719$, found 420.1718 .

$\mathrm{N}$-(2-Methylethyl-p-toluolsulfonamide)cantharidinimide (10j); $21 \%$ yield, m.p. $223-225^{\circ} \mathrm{C}$. IR (KBr): 1139 $\left(\mathrm{S}(=\mathrm{O})_{2}\right), 1397\left(\mathrm{~S}(=\mathrm{O})_{2}\right), 1691(\mathrm{C}=\mathrm{O}), 3244(\mathrm{NH}) \mathrm{cm}^{-1} ;[\alpha]_{\mathrm{D}}^{20}-5.6\left(c 0.5, \mathrm{CHCl}_{3}\right) ;{ }^{1} \mathrm{H}-\mathrm{NMR}$ (see Table 1); HRMS, calcd. for $\mathrm{C}_{20} \mathrm{H}_{26} \mathrm{~N}_{2} \mathrm{O}_{5} \mathrm{~S} 406.1562$, found 406.1560 .

$\mathrm{N}$-(2,2-Dimethylethyl-benzosulfonamide)cantharidinimide (10k): $22 \%$ yield, m.p. 155-156 ${ }^{\circ} \mathrm{C}$. IR (KBr): 1152 $\left(\mathrm{S}(=\mathrm{O})_{2}\right), 1400\left(\mathrm{~S}(=\mathrm{O})_{2}\right), 1695(\mathrm{C}=\mathrm{O}), 3253(\mathrm{NH}) \mathrm{cm}^{-1} ;{ }^{1} \mathrm{H}-\mathrm{NMR}: \delta 1.13(6 \mathrm{H}, \mathrm{s}), 1.23(6 \mathrm{H}, \mathrm{s}), 1.68-1.70$ $(2 \mathrm{H}, \mathrm{m}), 1.78-1.81(2 \mathrm{H}, \mathrm{m}), 3.52(2 \mathrm{H}, \mathrm{s}), 4.53(2 \mathrm{H}, \mathrm{t}, J=2.4 \mathrm{~Hz}), 5.88(1 \mathrm{H}, \mathrm{s}), 7.44(2 \mathrm{H}, \mathrm{t}, J=7.4 \mathrm{~Hz}), 7.49$ $(1 \mathrm{H}, \mathrm{t}, J=7.3,7.2 \mathrm{~Hz}), 7.84(2 \mathrm{H}, \mathrm{d}, J=7.5 \mathrm{~Hz}) ; \mathrm{HRMS}$, calcd. for $\mathrm{C}_{20} \mathrm{H}_{26} \mathrm{~N}_{2} \mathrm{O}_{5} \mathrm{~S} 406.1562$, found 406.1564 .

N-[2,2-Dimethylethylamino]-1-naphtharinimido-4-methylsulfanilamide (111): $10 \%$ yield, m.p. $247-248{ }^{\circ} \mathrm{C}$ (MeOH). IR (KBr): $1151\left(\mathrm{~S}(=\mathrm{O})_{2}\right), 1384\left(\mathrm{~S}(=\mathrm{O})_{2}\right), 1715(\mathrm{C}=\mathrm{O}), 3284 \mathrm{~cm}^{-1} ;{ }^{1} \mathrm{H}-\mathrm{NMR}\left(\mathrm{CDCl}_{3}, 500 \mathrm{~Hz}\right)$ : $\delta 1.28(6 \mathrm{H}, \mathrm{s}), 2.30(3 \mathrm{H}, \mathrm{s}), 3.89(2 \mathrm{H}, \mathrm{s}), 5.69(1 \mathrm{H}, \mathrm{s}), 7.10(2 \mathrm{H}, \mathrm{d}, J=8.1 \mathrm{~Hz}), 7.67(2 \mathrm{H}, \mathrm{d}, J=8.2 \mathrm{~Hz})$, $7.78(2 \mathrm{H}, \mathrm{dt}-\mathrm{like}, J=4.0,7.0,8.5 \mathrm{~Hz}), 7.88(2 \mathrm{H}, \mathrm{dt}-\mathrm{like}, J=3.1,5.3,6.8 \mathrm{~Hz})$; HRMS (EI, $80 \mathrm{ev})$, calcd. $\mathrm{C}_{19} \mathrm{H}_{20} \mathrm{~N}_{2} \mathrm{O}_{4} \mathrm{~S} 372.1144$, found 372.1143 .

N-[2-Methylethylamino]-1-naphtharinimido-4-methylsulfanilamide (11m): $73 \%$ yield, m.p. $200-202{ }^{\circ} \mathrm{C}$ (MeOH). IR (KBr): $1135\left(\mathrm{~S}(=\mathrm{O})_{2}\right), 1397\left(\mathrm{~S}(=\mathrm{O})_{2}\right), 1711(\mathrm{C}=\mathrm{O}), 3313 \mathrm{~cm}^{-1} ;{ }^{1} \mathrm{H}-\mathrm{NMR}: \delta 1.28(3 \mathrm{H}, \mathrm{d}$, $J=6.4 \mathrm{~Hz}), 2.09(3 \mathrm{H}, \mathrm{s}), 3.52(2 \mathrm{H}, \mathrm{dt}-\mathrm{like}, J=4.5,10.1,14.4 \mathrm{~Hz}), 3.68(1 \mathrm{H}, \mathrm{d}, J=7.6 \mathrm{~Hz}), 4.88(1 \mathrm{H}, \mathrm{d}$, $J=7.9 \mathrm{~Hz}), 6.86(2 \mathrm{H}, \mathrm{d}, J=8.0 \mathrm{~Hz}), 7.53(2 \mathrm{H}, \mathrm{d}, J=8.1 \mathrm{~Hz}), 7.71(4 \mathrm{H}, \mathrm{dt}-\mathrm{like}, J=5.4,9.0,10.2 \mathrm{~Hz}) ; \mathrm{HRMS}$

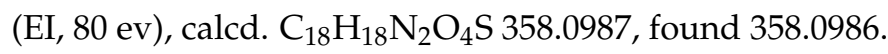

N-[2,2-Dimethylethylamino]-1-naphtharinimidosulfanilamide (11n): $34 \%$ yield, m.p. $202-203{ }^{\circ} \mathrm{C}(\mathrm{MeOH})$. IR (KBr): $1155\left(\mathrm{~S}(=\mathrm{O})_{2}\right), 1392\left(\mathrm{~S}(=\mathrm{O})_{2}\right), 1715(\mathrm{C}=\mathrm{O}), 3276 \mathrm{~cm}^{-1}$; ${ }^{1} \mathrm{H}-\mathrm{NMR}: \delta 1.29(6 \mathrm{H}, \mathrm{s}), 3.67(2 \mathrm{H}, \mathrm{s})$, $5.78(1 \mathrm{H}, \mathrm{s}), 7.31(2 \mathrm{H}, \mathrm{t}, J=7.7 \mathrm{~Hz}), 7.39(1 \mathrm{H}, \mathrm{t}, J=7.5 \mathrm{~Hz}), 7.76(2 \mathrm{H}, \mathrm{qd}, J=7.5,5.5,3.1,3.1 \mathrm{~Hz}), 7.81$ $(2 \mathrm{H}$, dd-like $J=7.7,1.1), 7.88(2 \mathrm{H}, \mathrm{dd}, J=3.1,5.4 \mathrm{~Hz})$; HRMS (EI, $80 \mathrm{ev)}$, calcd. $\mathrm{C}_{18} \mathrm{H}_{18} \mathrm{~N}_{2} \mathrm{O}_{4} \mathrm{~S} 358.0987$, found 358.0989 .

N-[3-(1,3-Dioxo-1,3,3a,4,7,7a-hexahydro-isoindol-2-yl)-2,2-dimethylethyl]-4-methyl-benzenesulfonamide (12o): 22\% yield, m.p. 133-134 ${ }^{\circ} \mathrm{C}(\mathrm{MeOH})$. IR (KBr): $1143\left(\mathrm{~S}(=\mathrm{O})_{2}\right), 1397\left(\mathrm{~S}(=\mathrm{O})_{2}\right), 1707(\mathrm{C}=\mathrm{O}), 3272(\mathrm{NH})$ $\mathrm{cm}^{-1}$; ${ }^{1} \mathrm{H}-\mathrm{NMR}: \delta 1.20(6 \mathrm{H}, \mathrm{s}), 2.22(2 \mathrm{H}, \mathrm{dd}, J=4.2,4.6 \mathrm{~Hz}), 2.40(3 \mathrm{H}, \mathrm{s}), 2.57(2 \mathrm{H}, \mathrm{dd}, J=1.6,1.6 \mathrm{~Hz})$, $3.10(2 \mathrm{H}, \mathrm{t}, J=2.8 \mathrm{~Hz}), 3.47(2 \mathrm{H}, \mathrm{s}), 5.79(2 \mathrm{H}, \mathrm{t}, J=3.1 \mathrm{~Hz}), 5.85(1 \mathrm{H}, \mathrm{s}), 7.25(2 \mathrm{H}, \mathrm{d}, J=9.4 \mathrm{~Hz}), 7.71$ $\left(2 \mathrm{H}, \mathrm{d}, J=8.2 \mathrm{~Hz}\right.$ ); HRMS (EI, $80 \mathrm{ev}$ ), calcd. $\mathrm{C}_{19} \mathrm{H}_{24} \mathrm{~N}_{2} \mathrm{O}_{4} \mathrm{~S} 376.1457$, found 376.1458 .

N-[3-(1,3-Dioxo-1,3,3a,4,7,7a-hexahydro-isoindol-2-yl)-2-methylethyl]-4-methyl-benzenesulfonamide (12p): 22\% yield, m.p. $145-146{ }^{\circ} \mathrm{C}(\mathrm{MeOH})$. IR (KBr): $1160\left(\mathrm{~S}(=\mathrm{O})_{2}\right), 1401\left(\mathrm{~S}(=\mathrm{O})_{2}\right), 1695(\mathrm{C}=\mathrm{O}), 3280(\mathrm{NH})$ $\mathrm{cm}^{-1} ;{ }^{1} \mathrm{H}-\mathrm{NMR}: \delta 1.00(3 \mathrm{H}, \mathrm{d}, J=6.6 \mathrm{~Hz}), 2.23(2 \mathrm{H}, \mathrm{dd}, J=5.3,15.5 \mathrm{~Hz}), 2.41(3 \mathrm{H}, \mathrm{s}), 2.54(2 \mathrm{H}, \mathrm{d}$, $J=18.1 \mathrm{~Hz}), 2.96(1 \mathrm{H}, \mathrm{dd}, J=2.7,2.9 \mathrm{~Hz}), 3.01(1 \mathrm{H}, \mathrm{dd}, J=2.7,10.7 \mathrm{~Hz}), 3.31(1 \mathrm{H}, \mathrm{dd}, J=3.7,13.7 \mathrm{~Hz})$, $3.49(1 \mathrm{H}, \mathrm{dt}-\mathrm{like}, J=5.9,9.4,10.9 \mathrm{~Hz}), 3.61(1 \mathrm{H}, \mathrm{s}), 4.78(1 \mathrm{H}, \mathrm{d}, J=8.4 \mathrm{~Hz}), 5.88(2 \mathrm{H}, \mathrm{t}, J=2.2 \mathrm{~Hz})$, 
$7.28(2 \mathrm{H}, \mathrm{d}, J=8.1 \mathrm{~Hz}), 7.70(2 \mathrm{H}, \mathrm{d}, J=8.1 \mathrm{~Hz})$; HRMS (EI, $80 \mathrm{ev})$, calcd. for $\mathrm{C}_{18} \mathrm{H}_{22} \mathrm{~N}_{2} \mathrm{O}_{4} \mathrm{~S} 362.1300$, found 362.1298 .

exo-N-Hydroxyl-7-oxabicyclo[2.2.1]hept-5-ene-2-phenylsulfonamido-1-methylethyl-2,3-dicarboximide (16q): $13 \%$ yield, m.p. $127-133^{\circ} \mathrm{C}(\mathrm{MeOH}) . \mathrm{IR}(\mathrm{KBr}): 1709(\mathrm{C}=\mathrm{O}) \mathrm{cm}^{-1},{ }^{1} \mathrm{H}-\mathrm{NMR}: \delta 1.34(3 \mathrm{H}, \mathrm{d}, J=3.3 \mathrm{~Hz})$, $2.71(2 \mathrm{H}, \mathrm{s}), 2.99-3.03\left(2 \mathrm{H}, \mathrm{m}, \mathrm{N}-\mathrm{CH}_{2}\right), 4.23-4.24(1 \mathrm{H}, \mathrm{m}, \mathrm{O}-\mathrm{CH}), 5.02(1 \mathrm{H}, \mathrm{s}), 5.19(1 \mathrm{H}, \mathrm{s}), 6.47(2 \mathrm{H}, \mathrm{s})$, 7.49-7.55 (3H, m), 7.85-7.86 (2H, m); FAB-MS m/z (rel. int. \%): $379[\mathrm{M}+\mathrm{H}]^{+}$(15), $311(48), 270(34)$, 70 (75), 56 (100).

exo-N-Hydroxyl-7-oxabicyclo[2.2.1]hept-5-ene-2-phenylsulfonamido-2-methylethyl-2,3-dicarboximide (16r): 9\% yield; $174-176{ }^{\circ} \mathrm{C}(\mathrm{MeOH})$; $\mathrm{IR}(\mathrm{KBr}): 1690(\mathrm{C}=\mathrm{O}) 3327 \mathrm{~cm}^{-1} ;{ }^{1} \mathrm{H}-\mathrm{NMR}: \delta 1.23(3 \mathrm{H}, \mathrm{d}, J=2.8 \mathrm{~Hz})$, $2.74(2 \mathrm{H}, \mathrm{dd}, J=5.4,7.3 \mathrm{~Hz}), 3.88(2 \mathrm{H}, \mathrm{td}, J=2.8,4.2,8.2 \mathrm{~Hz}), 3.51(1 \mathrm{H}, \mathrm{dd}, J=3.1,4.4 \mathrm{~Hz}), 5.24(2 \mathrm{H}, \mathrm{d}$, $J=4.9 \mathrm{~Hz}), 6.50(2 \mathrm{H}, \mathrm{s}), 7.49(3 \mathrm{H}, \mathrm{dt}-\mathrm{like}, J=5.8,6.0,11.3 \mathrm{~Hz}), 7.84(2 \mathrm{H}, \mathrm{dt}-\mathrm{like}, J=5.4,6.0,9.9 \mathrm{~Hz})$; FAB-MS m/z (rel. int. \%): 379 [M + H] ${ }^{+}$(3), 135 (70), 74 (75), 197 (35).

exo-N-Hydroxyl-7-oxabicyclo[2.2.1]hept-5-ene-2-[(2',5'-dichlorophenyl)sulfonamidoethyl]-2,3-dicarboximide (16s): $48 \%$ yield, m.p. $212-216{ }^{\circ} \mathrm{C}(\mathrm{MeOH}) . \mathrm{IR}(\mathrm{KBr}): 1780 \mathrm{~cm}^{-1},{ }^{1} \mathrm{H}-\mathrm{NMR}: \delta 2.77(2 \mathrm{H}, \mathrm{s}), 3.21(2 \mathrm{H}, \mathrm{dd}$, $J=5.5,10.1 \mathrm{~Hz}), 4.09(2 \mathrm{H}, \mathrm{t}, J=9.5 \mathrm{~Hz}), 5.25(2 \mathrm{H}, \mathrm{s}), 6.50(2 \mathrm{H}, \mathrm{s}), 6.40(1 \mathrm{H}, \mathrm{t}, 11.9 \mathrm{~Hz}), 7.44(2 \mathrm{H}, \mathrm{s})$, $8.04(1 \mathrm{H}, \mathrm{s})$; FAB-MS $m / z$ (rel. int. \%): $433[\mathrm{M}+\mathrm{H}]^{+}(4), 155$ (30), 365 (10); HRMS (FAB ${ }^{+}$), calcd. for $\mathrm{C}_{16} \mathrm{H}_{15} \mathrm{~N}_{2} \mathrm{O}_{6} \mathrm{Cl}_{2} \mathrm{~S} 433.0029$, found 433.0005 .

\subsection{Antineoplastic Bioassays}

\subsubsection{Cell Culture}

Media and sera for cell culture were purchased from Gibco/BRL (Grand Island, NY, USA). Most chemicals were purchased from Sigma Chemical (St. Louis, MO, USA). The human hepatoma (Hep3B) and myeloid leukemia (HL-60) cell lines were obtained from the American Type Culture Collection (ATCC; Rockville, MD, USA). The human breast cancer cell lines, MDA-MB-231 and MCF-7, were purchased from the cell bank of the National Health Research Institute (Miaoli, Taiwan). These cells were maintained as monolayers in Dulbecco's modified Eagle's medium (DMEM) containing 10\% heat-inactivated fetal bovine serum (FBS), 100 units $/ \mathrm{mL}$ penicillin, $100 \mu \mathrm{g} / \mathrm{mL}$ streptomycin, $100 \mu \mathrm{M}$ nonessential amino acids, and $1 \mathrm{mM}$ glutamine in a controlled atmosphere of $5 \% \mathrm{CO}_{2}$ and $95 \%$ air at $37^{\circ} \mathrm{C}$.

\subsubsection{MTT Assay for Cellular Viability}

Cells were seeded into 96-well plates and allowed to adhere for $24 \mathrm{~h}$ before drugs were introduced. Following a 48-h incubation, drugs and media were removed, and each well was treated with $100 \mu \mathrm{L}$ of $500 \mu \mathrm{g} / \mathrm{mL}$ 3-(4,5-dimethylthiazol-2-yl)-2,5-diphenyltetrazolium bromide (MTT) in culture medium. Following a 4-h incubation period to allow the metabolism of MTT by mitochondrial dehydrogenases of viable cells to form an insoluble formazan product, the plates were centrifuged at $450 \times \mathrm{g}$ for $10 \mathrm{~min}$, and supernatants were removed and replaced with $100 \mu \mathrm{L}$ DMSO. The plates were shaken to maximize solubilization of the formazan crystals. The absorbance, as a measure of viable cell numbers, was read the following day in a model MA310 automated EIA plate reader (Whittaker M. A. Bioproducts, Inc., Walkersville, MD, USA) at a wavelength of $550 \mathrm{~nm}$. IC 50 values were obtained by a linear regression analysis of the percent absorbance $v s$. the log of the drug concentration. It was previously shown that viable cell numbers are correlated with the optical density as determined in the MTT assay.

\section{Conclusions}

We synthesized cantharidinimide derivatives, $\mathbf{5 a}-\mathbf{h}$, and their analogues $\mathbf{1 0} \mathbf{i}-\mathbf{k}, \mathbf{1 1 1}-\mathbf{n}, \mathbf{1 2 0}-\mathbf{p}$, and $\mathbf{1 6 q}-\mathbf{s}$. The most potent cytostatic compound, $N$-cantharidinimido-sulfamethazine (5a), exhibited anti-HL-60 and anti-Hep3B cell activities. Two compounds, $5 \mathrm{~g}$ and $5 \mathbf{h}$, displayed slight effects on the Hep3B cell line, while the other compounds produced no response in these four cell lines. Although 
they exhibited very low effects on human carcinoma cells, we observed that these compounds contained sulfamoyl moieties. When studying the structure-activity relationship, we found that these compounds had very similar chemical structures to some diuretics, antidiabetic drugs, and hypoglycemic agents. Thus, their pharmacological effects should be tested in the future.

Acknowledgments: We thank Shwu-Huey Wang for help with the mass and NMR spectra and data calculation. The authors are indebted to Helmut Stamm (who recently passed away, School of Pharmacy, University of Heidelberg, Germany) for techniques of synthetic activated aziridines.

Author Contributions: Pen-Yuan Lin, Shiow-Yunn Sheu, and Mei-Hsiang Lin conceived and designed the experiments; Yun-Han Hsieh, Yi-Jing Lin, and Hsiao-Ling Chen performed the experiments and analyzed the data; Ling-Ling Chiang, Ing-Jy Tseng, and Ching-Tung Lin wrote the paper.

Conflicts of Interest: The authors declare no conflict of interest.

\section{References}

1. Moed, L.; Shwayder, T.A.; Chang, M.W. Cantharidin revisited: A blistering defense of an ancient medicine. Arch. Dermatol. 2001, 137, 1357-1360. [CrossRef] [PubMed]

2. Wang, G.S. Medical uses of Mylabris in ancient china and recent studies. J. Ethnopharmacol. 1989, 26, 147-162. [CrossRef]

3. Decker, R.H. Mechanism of acantholysis: The effect of cantharidin on oxidative phosphorylation. J. Investig. Dermatol. 1963, 42, 465-469. [CrossRef]

4. Li, Y.M.; Casida, J.E. Cantharidin-binding protein: Identification as protein phosphatase 2A. Proc. Natl. Acad. Sci. USA 1992, 89, 11867-11870. [CrossRef] [PubMed]

5. Ray, A.C.; Post, L.O.; Reagor, J.C. GC/MS confirmation of cantharidin toxicosis due to ingestion of blister beetles. Vet. Hum. Toxicol. 1980, 22, 398-399. [PubMed]

6. Sanchez-Barbudo, I.S.; Camarero, P.R.; Garcia-Montijano, M.; Mateo, R. Possible cantharidin poisoning of a great bustard (Otis tarda). Toxicon 2012, 59, 100-103. [CrossRef] [PubMed]

7. Honkanen, R.E. Cantharidin another natural toxin that inhibits the activity of serine/threonine protein phosphatases types 1 and 2A. FEBS Lett. 1993, 330, 283-286. [CrossRef]

8. Efferth, T.; Rauh, R.; Kahl, S.; Tomicic, M.; Bochzelt, H.; Tome, M.E.; Briehl, M.M.; Bauer, R.; Kaina, B. Molecular modes of action of cantharidin in tumor cells. Biochem. Pharmacol. 2005, 69, 811-818. [CrossRef] [PubMed]

9. Li, W.; Xie, L.; Chen, Z.; Zhu, Y.; Sun, Y.; Miao, Y.; Xu, Z.; Han, X. Cantharidin, a potent and selective PP2A inhibitor, induces an oxidative stress-independent growth inhibition of pancreatic cancer cells through G2/M cell-cycle arrest and apoptosis. Cancer Sci. 2010, 101, 1226-1233. [CrossRef] [PubMed]

10. Huang, W.W.; Ko, S.W.; Tsai, H.Y.; Chung, J.G.; Chiang, J.H.; Chen, K.T.; Chen, Y.C.; Chen, H.Y.; Chen, Y.F.; Yang, J.S. Cantharidin induces G2/M phase arrest and apoptosis in human colorectal cancer colo 205 cells through inhibition of CDK1 activity and caspase-dependent signaling pathways. Int. J. Oncol. 2011, 38, 1067-1073. [PubMed]

11. Hsia, T.C.; Yu, C.C.; Hsu, S.C.; Tang, N.Y.; Lu, H.F.; Huang, Y.P.; Wu, S.H.; Lin, J.G.; Chung, J.G. Cantharidin induces apoptosis of $\mathrm{H} 460$ human lung cancer cells through mitochondria-dependent pathways. Int. J. Oncol. 2014, 45, 245-254. [CrossRef] [PubMed]

12. Hsiao, Y.P.; Tsai, C.H.; Wu, P.P.; Hsu, S.C.; Liu, H.C.; Huang, Y.P.; Yang, J.H.; Chung, J.G. Cantharidin induces G2/M phase arrest by inhibition of $\mathrm{Cdc} 25 \mathrm{c}$ and Cyclin A and triggers apoptosis through reactive oxygen species and the mitochondria-dependent pathways of A375.S2 human melanoma cells. Int. J. Oncol. 2014, 45, 2393-2402. [CrossRef] [PubMed]

13. Su, C.C.; Liu, S.H.; Lee, K.I.; Huang, K.T.; Lu, T.H.; Fang, K.M.; Wu, C.C.; Yen, C.C.; Lai, C.H.; Su, Y.C.; et al. Cantharidin induces apoptosis through the Calcium/PKC-regulated endoplasmic reticulum stress pathway in human bladder cancer cells. Am. J. Chin. Med. 2015, 43, 581-600. [CrossRef] [PubMed]

14. Verma, A.K.; Prasad, S.B. Antitumor effect of blister beetles: An ethno-medicinal practice in Karbi community and its experimental evaluation against a murine malignant tumor model. J. Ethnopharmacol. 2013, 148, 869-879. [CrossRef] [PubMed] 
15. Xi, Y.; Garshott, D.M.; Brownell, A.L.; Yoo, G.H.; Lin, H.S.; Freeburg, T.L.; Yoo, N.G.; Kaufman, R.J.; Callaghan, M.U.; Fribley, A.M. Cantharidins induce ER stress and a terminal unfolded protein response in OSCC. J. Dent. Res. 2015, 94, 320-329. [CrossRef] [PubMed]

16. Zhang, S.Q. A study on antitumor chemotherapeutic agent-synthesis of $\mathrm{N}$-cantharidine derivatives. Acta Pharmacol. Sin. 1981, 16, 784-786.

17. Walter, W.G. Antitumor imide derivatives of 7-oxabicyclo[2.2.1]heptane-2,3-dimethyl-2,3-dicarboxylic acid. J. Pharmacol. Sci. 1989, 78, 66-67. [CrossRef]

18. Kok, S.H.L.; Chui, C.H.; Lam, W.S.; Chen, J.; Tan, J.C.O.; Lau, F.Y.; Cheng, G.Y.M.; Wong, R.S.M.; Chan, A.S.C. Induction of apoptosis on carcinoma cells by two synthetic cantharidin analogues. Int. J. Mol. Med. 2006, 17, 151-157. [CrossRef] [PubMed]

19. Fogh, J.; Fogh, J.M.; Orfeo, T. One hundred and twenty-seven cultured human tumor cell lines producing tumors in nude mice. J. Natl. Cancer Inst. 1977, 59, 221-226. [PubMed]

20. Tsauer, W.; Lin, J.G.; Lin, P.Y.; Hsu, F.L.; Chiang, H.C. The effects of cantharidin analogues on xanthine oxidase. Anticancer Res. 1997, 17, 2095-2098. [PubMed]

21. Essers, M.; Wibbeling, B.; Haufe, G. Synthesis of the first fluorinated cantharidin analogues. Tetrahedron Lett. 2001, 42, 5429-5433. [CrossRef]

22. McCluskey, A.; Keane, M.A.; Sim, A.T.R.; Walkom, C.C.; Young, D.J.; Bowyer, M.C.; Sakoff, J.A. The first two cantharidin analogues displaying PP1 selectivity. Bioorg. Med. Chem. Lett. 2002, 12, 391-393. [CrossRef]

23. Hart, M.E.; Chamberlin, R.; Walkom, C.; Sakoff, J.A.; McCluskey, A. Modified norcantharidins: Synthesis, protein phosphatases 1 and 2A inhibition, and anticancer activity. Bioorg. Med. Chem. Lett. 2004, 14, 1969-1973. [CrossRef] [PubMed]

24. Tatlock, J.H.; Linton, M.A.; Hou, X.J.; Kissinger, C.R.; Pelletier, L.A.; Showalter, R.E.; Tempczyk, A.; Villafranca, J.E. Structure-based design of novel calcineurin (PP2B) inhibitors. Bioorg. Med. Chem. Lett. 1997, 7, 1007-1012. [CrossRef]

25. Tarleton, M.; Gilbert, J.; Sakoff, J.A.; McCluskey, A. Synthesis and anticancer activity of a series of norcantharidin analogues. Eur. J. Med. Chem. 2012, 54, 573-581. [CrossRef] [PubMed]

26. Puerto Galvis, C.E.; Vargas Mendez, L.Y.; Kouznetsov, V.V. Cantharidin-based small molecules as potential therapeutic agents. Chem. Biol. Drug Des. 2013, 82, 477-499. [CrossRef] [PubMed]

27. Luzina, E.L.; Popov, A.V. Synthesis and anticancer activity evaluation of 3,4-mono- and bicyclosubstituted $\mathrm{N}$-(het)aryl trifluoromethyl succinimides. J. Fluorine Chem. 2014, 168, 121-127. [CrossRef] [PubMed]

28. Lin, P.Y.; Shi, S.J.; Shu, H.L.; Chen, H.C.; Lin, C.C.; Liu, P.C.; Wang, L.F. A simple procedure for preparation of $N$-thiazolyl and $N$-thiadiazolylcantharidinimides and evalution of their cytotoxicities against human hepatocellular carcinoma cell. Bioorg. Chem. 2000, 28, 266-272.

29. Lin, L.H.; Huang, H.S.; Lin, C.C.; Lee, L.W.; Lin, P.Y. Effects of cantharidins on human carcinoma cells. Chem. Pharm. Bull. 2004, 52, 855-857. [CrossRef] [PubMed]

30. Tseng, I.J.; Sheu, S.Y.; Chen, Y.T.; Huang, C.Y.; Lin, C.T.; Lin, P.Y. A simple procedure for preparation of $N$-thiazol, thiadiazol, pyridyl and sulfanylamidocantharidinimimes analogues and evaluation of their cytotoxicities against human HL-60, MCF7, Neuro-2a and A549 carcinoma cell. Chem. Pharm. Bull. 2012, 60, 1453-1457. [CrossRef] [PubMed]

31. Tseng, I.J.; Sheu, S.Y.; Lin, P.Y.; Lee, J.A.; Ou, K.L.; Lee, L.W. Synthesis and evaluation of cantharidinimides on human cancer cells. J. Exp. Clin. Med. 2012, 4, 280-283. [CrossRef]

32. Yej, C.B.; Lin, P.Y.; Huang, J.M.; Su, C.J.; Yeh, Y.T.; Yang, S.F.; Chou, M.C. Study on synthesis of thalidomide analogues and their bioactivities: Inhibition on iNOS pathway cytotoxic effects. Med. Chem. Res. 2012, 21, 953-963.

33. Tseng, I.J.; Lin, P.Y.; Sheu, S.Y.; Tung, W.N.; Lin, C.T.; Lin, M.H. Characterization of novel aminobenzylcantharidinimides and their effects on NO induction. J. Chin. Chem. Soc. 2015, 62, 59-63. [CrossRef]

34. Lin, P.Y.; Bellos, K.; Stamm, H.; Onistschenko, A. Acylated 2,2-dimethylaziridin: Regioselectivity of ring opening thiophenolate; borderline $\mathrm{S}_{\mathrm{N}} 2$ sue to planarization of nitrogen pyramid. Tetrahedron 1992, 48, 2359-2372. [CrossRef]

35. Bellos, K.; Stamm, H.; Speth, D. N-S Cleavage is faster than homolytic ring opening in single-electron transfer to some $\mathrm{N}$-sulfonylaziridines. Competition between $\mathrm{S}_{\mathrm{N}} 2$ and SET. J. Org. Chem. 1991, 56, 6846-6849. [CrossRef] 
36. Lin, P.Y.; Weiee, R.; Werry, J.; Falkenstein, R.; Stamm, H. Neglected aspects of anthracenide (anthracenidyl) chemistry reactions of two N-benzoylaziridines. J. Prakt. Chem. 2000, 342, 153-161. [CrossRef]

37. Kadioglu, O.; Kermani, N.S.; Kelter, G.; Schumacher, U.; Fiebig, H.H.; Greten, H.J.; Efferth, T. Pharmacogenomics of cantharidin in tumor cells. Biochem. Pharmacol. 2014, 87, 399-409. [CrossRef] [PubMed]

38. Wosikowski, K.; Schuurhuis, D.; Johnson, K.; Paull, K.D.; Myers, T.G.; Weinstein, J.N.; Bates, S.E. Identification of epidermal growth factor receptor and c-erbB2 pathway inhibitors by correlation with gene expression patterns. J. Natl. Cancer Inst. 1997, 89, 1505-1515. [CrossRef] [PubMed]

39. Luzina, E.L.; Popov, A.V. Synthesis and anticancer activity of $N$-bis(trifluoromethyl)alkyl- $N^{\prime}$-thiazolyl and $N$-bis(trifluoromethyl)alkyl- $N^{\prime}$-benzothiazolyl ureas. Eur. J. Med. Chem. 2009, 44, 4944-4953. [CrossRef] [PubMed]

40. Luzina, E.L.; Popov, A.V. Anticancer activity of $N$-bis(trifluoromethyl)alkyl- $N^{\prime}$-(polychlorophenyl) and $N^{\prime}$-(1,2,4-triazolyl) ureas. Eur. J. Med. Chem. 2010, 45, 5507-5512. [CrossRef] [PubMed]

41. Luzina, E.L.; Popov, A.V. Synthesis, evaluation of anticancer activity and COMPARE analysis of $N$-bis(trifluoromethyl)alkyl- $N^{\prime}$-substituted ureas with pharmacophoric moieties. Eur. J. Med. Chem. 2012, 53, 364-373. [CrossRef] [PubMed]

42. Luzina, E.L.; Popov, A.V. Synthesis of 3,3,3-trifluoroethyl isocyanate, carbamate and ureas. Anticancer activity evaluation of $N$-(3,3,3-trifluoroethyl)- $N^{\prime}$-substituted ureas. J. Fluorine Chem. 2015, 176, 82-88. [CrossRef] [PubMed]

Sample Availability: Samples of the compounds 10i-k, 111-n, 12o-p, and 16q-s are available from the authors.

(C) 2016 by the authors; licensee MDPI, Basel, Switzerland. This article is an open access article distributed under the terms and conditions of the Creative Commons by Attribution (CC-BY) license (http://creativecommons.org/licenses/by/4.0/). 